\title{
Competencias comunicativas y digitales impulsadas en escuelas rurales elaborando digital storytelling
}

\author{
María Esther del Moral Pérez, Lourdes Villalustre Martínez, María Rosario Neira Piñeiro \\ Departamento de Ciencias de la Educación. Universidad de Oviedo
}

\begin{abstract}
RESUMEN
Los digital storytelling (DST) constituyen una práctica narrativa innovadora que combina múltiples lenguajes, potenciando el aprendizaje lecto-escritor utilizando herramientas digitales. Esta investigación, derivada del Proyecto de Innovación CINEMA -Convenio de colaboración Universidad de Oviedo-Consejería de Educación 2014-15-, centrado en la creación de DST, analiza el cambio observado por el profesorado en el desarrollo de las competencias comunicativa y digital alcanzado por 282 alumnos procedentes de aulas multinivel de escuelas rurales, utilizando un instrumento ad hoc con 15 indicadores. Los resultados evidencian que, en la competencia comunicativa, las alumnas de Educación Infantil destacan por su expresión (vocabulario y pronunciación), comprensión de nociones espacio-temporales, muestran más interés y participan en la creación del relato colaborativamente. En Primaria todos mejoran cualitativamente, especialmente los cursos superiores, al elaborar composiciones escritas para los DST (vocabulario, ortografía, puntuación, tiempos verbales), crear un guión con personajes y escenarios coherentes y explicar la intención de su relato. Además, las alumnas de Educación Infantil destacan al utilizar mejor los medios tecnológicos que sus compañeros. Asimismo, los cursos superiores de primaria la desarrollan más, dada la complejidad progresiva de las herramientas empleadas para elaborar los DST. En conclusión, la elaboración de DST fomenta la capacidad de expresión y comunicación de los escolares.
\end{abstract}

Palabras Clave: digital storytelling, competencia comunicativa, competencia digital, escuela rural, educación primaria.

\section{Communicative and digital competences promoted in rural schools with digital storytelling}

\begin{abstract}
Digital storytelling (DST) is a narrative innovative practice that combines several languages and promotes literacy with digital tools. This research comes from CINEMA project, carried out in the framework of collaboration agreement between the Department of Education and the University of Oviedo in the year academic year 2014/2015. The paper analyses the development of communicative and digital competences in 282 students in multi-grade classrooms of rural schools, by means of a tool created ad hoc, with 15 indicators. Regarding communicative competence, the results reveal that preschool girls stand out in expression (vocabulary and pronunciation) and understanding of space and temporal notions, show more interest and participate more in the collaborative creation of the story. In Primary Education, all of them progress qualitatively, especially the older children, when writing for DST (vocabulary, spelling, punctuation, verb tenses), creating a screenplay with coherent characters and settings, and explaining the purpose of their story. Regarding digital competence, preschool girls are better than their male classmates in using technological media. Besides, in the latest stages of Primary Education digital competence is more fostered, due to the ongoing complexity of the tools used to create the DST. In conclusion, DST creation fosters expression and communication abilities in schoolchildren.
\end{abstract}

Keywords: digital storytelling, communicative competence, digital competence, rural school, primary education.

\section{Introducción}

La realización de prácticas formativas apoyadas en herramientas tecnológicas hace imprescindible que las escuelas cuenten no solo con unas mínimas infraestructuras y dotaciones básicas con acceso a Internet, sino también con un profesorado cualificado para dinamizar e impulsar proyectos innovadores y que, además, posea las competencias digitales necesarias para garantizar su éxito (De Pablos, Colás y González, 2010). En este sentido, algunos programas institucionales, como el extinto pro- 
grama Escuela 2.0, han ayudado a favorecer la cultura innovadora en los centros escolares apuntalando estos dos frentes, siendo el profesorado la pieza clave para promover proyectos de calidad (Area, 2010; Valverde, Garrido, y Sosa, 2010). Para las escuelas rurales, estas inversiones en recursos y formación han contribuido a minimizar la brecha que las separa de las urbanas, gracias al afán de los docentes por aprovechar las ventajas ligadas a las nuevas tecnologías para dar respuesta a las demandas del medio donde se insertan (Del Moral, Villalustre y Neira, 2014a, 2014b).

Así pues, a pesar de que son numerosas las experiencias formativas que apuestan por el uso de las Tecnologías de la Información y la Comunicación (TIC) para optimizar el proceso de enseñanza-aprendizaje y favorecer la adquisición de diferentes habilidades, aquí se pondrá especial énfasis en estudiar aquellas prácticas docentes basadas en la creación colaborativa de relatos digitales o digital storytelling (DST), por considerarla una interesante estrategia didáctica que los docentes pueden implementar en sus aulas para promover habilidades diversas (Robin, 2008; Skouge y Rao, 2009).

A este respecto, existen investigaciones que resaltan las oportunidades brindadas por los DST para facilitar la adquisición de competencias comunicativas y narrativas (Dettori y Paiva, 2009; Ohler, 2013), relacionadas con el aprendizaje de la lecto-escritura, junto con otras de carácter digital propias de la sociedad del siglo XXI (Malita y Martin, 2010) vinculadas al manejo de herramientas tecnológicas. Además, Figg, McCartney y Gonsoulin (2010) demuestran la relación del diseño colaborativo de DST con un incremento en el rendimiento académico de los estudiantes, previa sistematización del proceso y de las tareas a desarrollar, adoptando una metodología interdisciplinar (Frazel, 2010).

En este sentido, el proyecto CINEMA -avalado por la Consejería de Educación- sirvió de catalizador para consolidar prácticas formativas innovadoras en varias escuelas rurales asturianas, involucrando a 50 docentes que apuestan por la utilización de herramientas digitales para crear narraciones colaborativas implicando a todo el alumnado. Para ello, se conformó una comunidad de práctica que dispensó a los docentes la formación y los recursos necesarios para llevarlo a cabo. Concretamente, esta investigación pretende analizar como la elaboración colaborativa de DST en aulas de Educación Infantil y Primaria de estas escuelas de contextos rurales -caracterizadas por ser aulas multigrado, donde convergen niños y niñas de varias edades- ha contribuido al desarrollo de sus competencias comunicativas y digitales.

\section{Digital storytelling: recursos para la nueva alfabetización y desarrollo de competencias}

La propuesta formativa basada en la creación de digital storytelling toma como referente el modelo teórico de alfabetización para formar a los ciudadanos de la era digital -propuesto por Area y Ribeiro (2012)-, dado que procura fortalecerlos desde su doble dimensión. Por un lado, se contempla la alfabetización genérica para el uso y explotación de recursos de la web 2.0, y, por otro, el impulso de las competencias ligadas al aprendizaje: las instrumentales, como el manejo de recursos tecnológicos; las de tipo cognitivo-intelectual, relacionadas con la búsqueda, selección, organización y análisis crítico de la información; las socio-comunicacionales, para la elaboración de las propias producciones digitales y las denominadas emocionales y axiológicas, subrayando el componente emocional y ético plasmado en dichas producciones.

Así pues, estas nuevas fórmulas narrativas que combinan diferentes lenguajes, integrando información multiformato (tex- to, audio, vídeo, imágenes, etc.) de forma coherente y atractiva, se constituyen en actividades de aprendizaje significativas que promueven la nueva alfabetización en las aulas de la era digital, así como el desarrollo de numerosas competencias, tales como la digital, la narrativa, la creativa, etc. (Villalustre y Del Moral, 2014). Se trata de unas prácticas formativas claves para impulsar los talentos y habilidades de los estudiantes de forma transversal (hler, 2013), que permiten desarrollar las competencias inherentes a la era digital ( $\mathrm{Heo}, 2009$; Robin, 2008) al aprovechar las ventajas que ofrecen las aplicaciones de la web 2.0 (Lowenthal, 2009). Además, en algunos casos sirven de vehículo para el aprendizaje de sencillos lenguajes de programación, al facilitar la organización de secuencias lógicas de acontecimientos y adaptar la composición narrativa al pensamiento computacional (Burke y Kafai, 2012).

Indudablemente, el proceso de elaboración de los relatos digitales implica el desarrollo de la competencia comunicativa, vinculada a la creación de narraciones a partir de imágenes, fotos, ilustraciones, fragmentos de vídeos, etc., cargadas de contenido e impregnadas de valores culturales (Dobson, 2005; Gazarian, 2010), que pueden venir locutadas y acompañadas con bandas sonoras y/o sonidos onomatopéyicos para dotarlos de un valor estético añadido. Además, el diseño de estos relatos requiere el uso de la lengua oral y escrita, lo que supone la puesta en práctica de diferentes destrezas. Todo ello lo convierte en una compleja tarea que activa las habilidades creativas (Lambert, 2010), subrayando la capacidad expresiva de los recursos tecnológicos (Rodríguez-Illera y Escofet, 2006).

\subsection{Competencias comunicativas promovidas con los relatos digitales}

La competencia comunicativa, referida a la capacidad de los hablantes de una lengua para comunicarse eficazmente en diferentes contextos (Gumperz y Hymes, 1972, citado en Lomas, 2015), posee gran relevancia tanto en el ámbito educativo como en la vida cotidiana, y como tal es incluida entre las competencias clave propuestas por el Parlamento Europeo y Consejo (2006), aunque desglosada en dos: comunicación en la lengua materna y comunicación en lenguas extranjeras. Ambas suponen la capacidad de "comunicarse de forma oral y escrita en múltiples situaciones comunicativas y para controlar y adaptar su propia comunicación a los requisitos de la situación" Parlamento Europeo y Consejo $(2006$, p. 14), así como la posesión de habilidades específicas referidas al manejo de distintos tipos de textos, la búsqueda y procesamiento de información y la comprensión intercultural. Asimismo, la competencia comunicativa es una de las competencias clave del currículo actual español, con la denominación "comunicación lingüística".

La competencia comunicativa incluye diferentes dimensiones para las que existen diversas propuestas de clasificación -como la de Canale (1983), la del Marco Común Europeo (Instituto Cervantes, 2002)- o la recogida en el currículo actual (마en ECD/65/2015). Todas coinciden en identificar una dimensión lingüística o gramatical, referida al conocimiento y uso del código de la lengua, que incluye el dominio de los diferentes subsistemas (fonológico, ortográfico, léxico, etc.). Dado que la creación de relatos digitales requiere la utilización de la lengua oral y escrita en distintas tareas, todos los aspectos de la dimensión lingüística son activados a lo largo del proceso.

La capacidad de comunicarse eficazmente precisa también el conocimiento y control de códigos sociales y culturales que intervienen en las comunicaciones, así como el manejo de la dimensión la pragmática, referida al uso de la lengua en su contexto. 
Ésta última da cabida a la competencia discursiva -referida al conocimiento de los códigos de los diferentes modelos textuales y a la capacidad de producir textos coherentes y cohesionados- y a la dimensión pragmática -que incluye los aspectos funcionales de la lengua-, dimensiones todas ellas activadas en las tareas comunicativas implicadas en la creación de DST.

\subsection{Competencias digitales inherentes a la narración digital}

La competencia digital es una de las ocho enunciadas por el Parlamento Europeo y Consejo (2006), que deben desarrollarse en las diferentes etapas de la enseñanza obligatoria. Ésta se orienta a proporcionar las habilidades y capacidades necesarias para conocer y manejar las tecnologías digitales y hacer frente a los desafíos que plantean.

El sistema educativo debe aprovechar e integrar de forma eficaz las oportunidades que ofrecen las tecnologías, pues no en vano es uno de los objetivos prioritarios del "Marco estratégico europeo de educación y formación-ET 2020" (ITE, 2011). No obstante, Mihailidis y Cohen (2013) consideran que el desarrollo de la competencia digital en las aulas está condicionado por la formación de los docentes y por el equipamiento disponible en los centros educativos. En este sentido, las escuelas rurales tienen mayores dificultades para desarrollar esta competencia debido a que no siempre disponen de las infraestructuras tecnológicas necesarias (Del Moral, Villalustre y Neira, 2014b), aunque estas carencias son suplidas con iniciativas innovadoras, como el diseño de digital storytelling, que permiten reforzar las habilidades y capacidades asociadas a la competencia digital (Banaszewski, 2002).

En el desarrollo de esta competencia inciden numerosos aspectos metodológicos, plasmados en el currículum transversalmente, y supone la base para impulsar otras competencias clave, como la comunicativa o lingüística, pues se relaciona con ciertas habilidades básicas que abarcan desde la búsqueda y transformación de la información hasta la producción de recursos expresivos utilizando diferentes lenguajes y técnicas (Lai, 2011). En ella convergen tres tipos de alfabetización: informacional, tecnológica y mediática, convirtiéndola en una competencia básica y fundamental del currículum escolar (Guo, Dobson y Petrina, 2008).

La competencia digital se sustenta en la adquisición y desarrollo de habilidades para buscar, obtener, procesar y comunicar información, así como para transformarla en conocimiento (Hatlevik y Christophersen, 2013). Está asociada con la habilidad para conocer y manejar lenguajes, técnicas y estrategias propias de los diferentes soportes, tales como el audiovisual, digital o multimedia (Krumsvik, 2014). Por su parte, Calvani, Ranieri y Fini (2010) la relacionan con la habilidad para hacer un uso seguro y crítico de las tecnologías, valorando sus fortalezas y debilidades para la interacción y participación social desde una actitud crítica, activa y comprometida. También requiere de habilidades para la resolución de problemas, así como para la evaluación, selección e intercambio de nuevas fuentes de información para acometer tareas colaborativas. Janssen, Stoyanov, Ferrari, Punie, Pannekeet y Sloep (2013) consideran que contribuye a potenciar diferentes registros y lenguajes de comunicación mediante el uso interactivo de diversas herramientas y dispositivos, así como el incremento del pensamiento reflexivo, la creatividad y la innovación.

Con todo, la investigación que se presenta intenta analizar si la práctica de creación colaborativa de relatos digitales permite desarrollar tanto la competencia comunicativa como la digital en los escolares.

\section{Método}

CINEMA es un proyecto de innovación centrado en el diseño colaborativo de relatos digitales, fruto del Convenio de Colaboración entre la Universidad de Oviedo y la Consejería de Educación asturiana (curso 2014-15). Se desarrolló en seis escuelas rurales que contaban con la dotación tecnológica del Programa Escuela 2.0., con el objetivo principal de potenciar la adquisición de las competencias comunicativa y digital en los escolares participantes.

En un primer momento, se buscó dotar de la cualificación y el apoyo necesario al profesorado implicado ( $\mathrm{N}=50$ docentes), mediante la creación de una comunidad de práctica virtual que integrara a los docentes, para: a) ofrecerles pautas procedimentales básicas para la realización de relatos, recursos y herramientas digitales accesibles on line para su ejecución, tutoriales, ejemplos con diferentes técnicas, etc.; b) fomentar la comunicación y el intercambio de experiencias, y c) facilitar el diseño consensuado de instrumentos, a partir de indicadores cualitativos que les permitiera constatar los progresos alcanzados por los alumnos. Posteriormente, una vez concluida la experiencia innovadora de narración digital, de la que se derivaron catorce relatos, los docentes valoraron el cambio observado durante su desarrollo en el nivel competencial de los alumnos, efectuando una evaluación individualizada de cada uno de ellos.

Esta investigación adopta una metodología descriptivo-cuantitativa para analizar los datos recabados mediante un instrumento de evaluación de las competencias comunicativa y digital. La necesidad de disponer de una herramienta adaptada al proyecto, que resultase eficaz para la evaluación por parte del profesorado participante, hizo necesario la creación de un instrumento diseñado ad hoc, compuesto de 15 indicadores. Para ello, se partió, tal como se explica más adelante, de las indicaciones para la evaluación de la competencia comunicativa recogidas en el Marco Común Europeo de Referencia para las lenguas (Instituto Cervantes, 2002) así como de las aportaciones del ITE (2011), Hatlevik y Christophersen (2013) y Krumsvik (2014) para medir la competencia digital. Ello permitió determinar una serie de indicadores, adaptados a las características del alumnado y de la tarea, que fue consensuado con los docentes de infantil y primaria -junto a los especialistas e investigadores universitarios- y validado a través del método Delphi (Clayton, 1997), teniendo para ello en cuenta los criterios establecidos por Hung, Altschuld y Lee (2008), configurando así el instrumento final (Del Moral, Villalustre y Neira, 2016). Dicho instrumento se utilizó posteriormente para que el profesorado evaluara individualmente a los alumnos de Educación Infantil y Primaria que participaron en el proyecto.

\section{Objetivo}

La finalidad de esta investigación es analizar en qué medida la elaboración colaborativa de DST en aulas de Educación Infantil y Primaria de las escuelas rurales -implicadas en el proyecto CINEMA-, ha contribuido a potenciar las competencias comunicativa y digital de los escolares participantes.

\section{Muestra}

En el proyecto CINEMA participaron 50 maestros de Educación Infantil y Primaria, quienes impulsaron la creación de los relatos y llevaron a cabo la evaluación individualizada de cada alumno. Así, la muestra total participante en el proyecto fue de 282 estudiantes de Educación Infantil y Primaria proce- 
dentes de escuelas ubicadas en contextos rurales, -en su mayoría caracterizadas por ser aulas multigrado aglutinando a escolares de varias edades-, donde el $47 \%$ eran alumnas y el 53\% restante alumnos. Un análisis más detallado en función de la etapa educativa revela que en Educación Primaria hubo una participación susceptiblemente mayor de alumnos en el proyecto (54,7\%), mientras que en Educación Infantil las alumnas tuvieron mayor presencia $(50,6 \%)$.

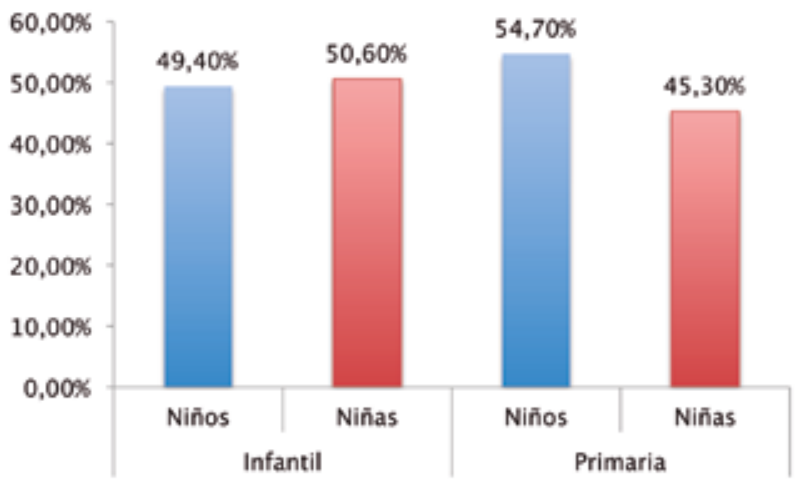

Gráfico 1. Distribución porcentual de la totalidad de la muestra según sexo y etapa educativa

Por otro lado, en Educación Infantil más de la mitad de los niños y niñas participantes (52\%) pertenecían al último curso de esta etapa educativa, mientras que el resto se reparte de forma equilibrada entre $1^{\mathrm{O}}$ y $2^{\mathrm{o}}$ de Infantil con porcentajes que oscilan entre el $23,5 \%$ y $24,5 \%$.

En Educación Primaria, la distribución de los sujetos en los diferentes cursos varía sustancialmente, apreciándose que en $5^{\mathrm{o}}$ y $6^{\mathrm{o}}(23,3 \%$ y $23,9 \%$ respectivamente) se concentra el mayor número de estudiantes que participaron en el proyecto, en contraposición con aquellos de $1^{\mathrm{O}}$ y $2^{\mathrm{o}}$ de educación primaria, cuya presencia ha sido más discreta ( $8 \%$ respectivamente). Para facilitar los análisis estadísticos, se procede a agrupar los seis cursos de primaria en una nueva variable denominada "nivel", definida en tres categorías: 1) nivel inicial, que aglutina a los escolares de $1^{\circ}$ y $2^{\circ}$; 2) nivel medio, que agrupa al alumnado de $3^{\circ}$ y $4^{\circ}$; y 3 ) nivel superior, que reúne a los estudiantes de $5^{\circ}$ y $6^{\circ}$ de primaria.

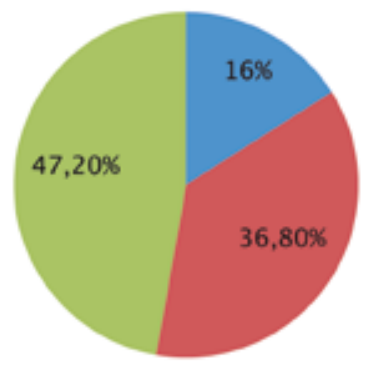

"Nivel Inicial = Nivel Medio $=$ Nivel Superior

Gráfico 2. Distribución porcentual del alumnado de Primaria según la variable "nivel"

En función de dicho agrupamiento se observa (gráfico 2) que el $16 \%$ de los alumnos de educación primaria pertenecen al nivel inicial, seguidos por el 36,8\%, que se concentran en el nivel medio, mientras que el $47,2 \%$ de los escolares de educación primaria se encuentran en el nivel superior.

\section{Instrumento}

Los 50 docentes implicados en el proyecto diseñaron de forma consensuada, junto con los investigadores, el instrumento -validado por el método Delphi y publicado en Del Moral, Villalustre y Neira (2016)- para evaluar las competencias comunicativa y digital desarrolladas por sus alumnos con la realización de los relatos digitales. Este instrumento, integrado por 15 indicadores - 10 relativos a la competencia comunicativa y 5 a la digital-, permitió sistematizar y evaluar individualmente el cambio observado en el nivel competencial de cada alumno tras participar en la creación de relatos digitales. Dichos indicadores se adaptaron a las particularidades madurativas de los alumnos de Infantil y de Primaria, pues implican matices diferenciales para su evaluación, utilizando escalas tipo Likert con cinco opciones de respuesta ( $1=$ poco; $2=$ algo; $3=$ bastante; $4=$ mucho; $5=$ no procede) en ambos casos.

a) Evaluación de la competencia comunicativa adquirida:

Aunque para la evaluación de la competencia comunicativa se partió de las orientaciones recogidas en el Marco (Instituto Cervantes, 2002), el instrumento diseñado al efecto se ajustó a las características de esta experiencia, considerando los aspectos ligados al proceso de diseño y creación de relatos digitales y, adaptándolo a las capacidades exigibles al alumnado de Infantil y Primaria. En Educación Infantil se seleccionaron aspectos relativos a la competencia lingüística, funcional y discursiva, referida esta última a la comprensión y creación de narraciones. Además, se dio prioridad a la comunicación oral, aunque contemplando también la iniciación en la lecto-escritura. En Educación Primaria se tuvieron en cuenta aspectos relativos a la competencia lingüística (oral y escrita), funcional (adecuación a la intencionalidad), discursiva (referida a la narración y a producción de textos coherentes y cohesionados destinados a la creación audiovisual) y, en menor medida, sociolingüística (registro).

\section{b) Evaluación de la competencia digital desarrollada}

Por otro lado, otros 5 indicadores -extraídos de las aportaciones del_ITE (2011), Hatlevik y Christophersen (2013) y Krumsvik (2014)-, sirvieron para medir la competencia digital del alumnado de Infantil tras su participación en el proyecto, abordando aspectos relativos tanto al uso y manejo de las herramientas tecnológicas, como la capacidad expresiva y creativa de los medios digitales. Por su parte, para evaluar a los estudiantes de primaria se tuvieron en cuenta, principalmente, aspectos relativos a los conocimientos sobre el lenguaje audiovisual, así como la correcta utilización de programas para la creación y edición de relatos.

\section{Procedimiento}

CINEMA se organizó en torno a una comunidad de práctica virtual que proporcionó formación básica, acceso a herramientas digitales y ejemplos a los docentes participantes, facilitando tanto el intercambio de experiencias como la elaboración consensuada de un instrumento cualitativo que les ayudó a medir el cambio observado en el nivel de competencia comunicativa y digital de los alumnos derivado de su participación en el proyecto. 


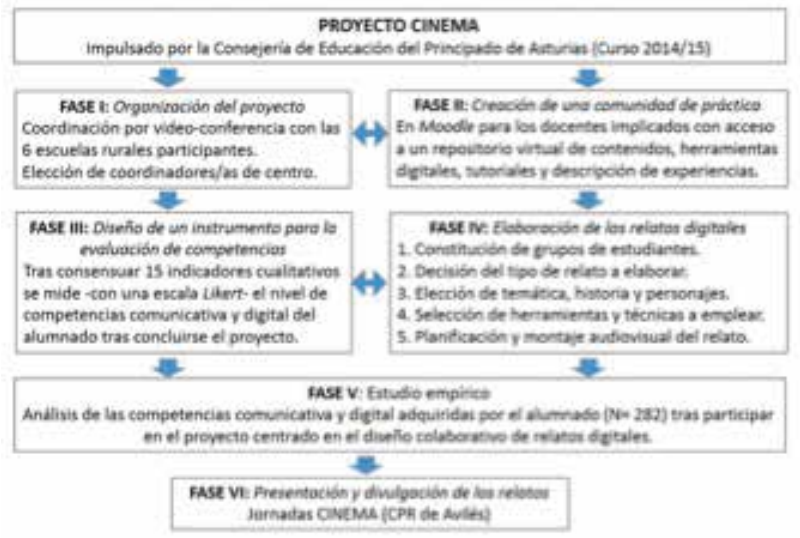

Figura 1. Fases del Proyecto CINEMA

Finalizada la fase de creación de los relatos, el profesorado valoró individualmente el desarrollo de las competencias comunicativas y digitales del alumnado. Los datos se sometieron a análisis estadístico para comparar las medias -en función de las variables "sexo", "curso" (para Infantil) y "nivel" (para Primaria). Los contrastes posteriores se obtuvieron a partir del ANOVA. Los estadísticos se estimaron con el $95 \%$ de confiabilidad, utilizando el paquete estadístico SPSS (v.22).

\section{Resultados}

\subsection{Evaluación de la competencia comunicativa alcanzada con los relatos digitales}

\section{Educación Infantil}

Los diez indicadores ligados a la elaboración de los DST que sirvieron para valorar el nivel de competencia comunicativa desarrollado por el alumnado de Educación Infantil revelan, -a juicio del profesorado implicado-, que esta práctica formativa ha proporcionado un escenario motivador para ejercitar las habilidades lingüísticas, relacionadas tanto con la capacidad para comprender mensajes de carácter textual o audiovisual, como para elaborar los suyos propios con el apoyo del profesorado (Tabla 1).
Sin duda, el nivel de motivación del 75\% de los alumnos por la actividad propuesta queda reflejado en el alto interés manifestado por las aplicaciones digitales en tanto favorecedoras del aprendizaje lecto-escritor, puesto que un $45,7 \%$ presenta mucho interés y un $29,6 \%$ bastante. Estos datos se relacionan de forma directa con el alto nivel de comprensión manifestado por las narraciones audiovisuales sencillas presentadas $(82,7 \%)$, siendo para el $53,1 \%$ mucha y para el $29,6 \%$ bastante. Son formatos atractivos con los que están familiarizados, conocen su estructura y entienden fácilmente las historias narradas.

Otra de las habilidades implicadas en esta actividad es la capacidad para compartir y expresar sus ideas oralmente, a pesar de que hay distintos niveles de madurez en Infantil: 30,9\% medio, $34,6 \%$ alto y $33,3 \%$ muy alto, pues están superando la etapa egocéntrica y abriéndose a la comunicación con otros. Sin embargo, un $68 \%$ aglutina a los que la desarrollaron mucho y bastante esta habilidad. El 35,8\% refleja un nivel alto y el 34,6\% muy alto en su capacidad para expresarse clara y coherentemente, el 70,4\% utiliza vocabulario acorde a su edad.

Por otro lado, la mayoría de los relatos incluía locuciones a modo de voz en off que acompañaban las imágenes que ilustraban las historias, lo que -según los docentes- les permitió ejercitar la pronunciación y entonación de breves textos, a un $34,6 \%$ bastante y a otro $34,6 \%$ mucho (Tabla 1 ). Ligado a ello, se constata el nivel de reconocimiento y utilización de los elementos propios del lenguaje escrito alcanzado por ellos, teniendo en cuenta que se hallan en un periodo de iniciación e identificación de palabras básicas, para un $35,8 \%$ es alto y para un $28,4 \%$ muy alto.

Si bien en las aulas de Infantil es recurrente la utilización de narraciones orales con fines educativos, esta actividad supuso que el $44,4 \%$ haya desarrollado bastante su habilidad para narrar una historia oralmente con ayuda de los docentes, y casi un $30 \%$ lo ha hecho mucho. Sin embargo, su participación en la creación colaborativa del relato ha sido desigual: mientras un aproximado $60 \%$ se implicó bastante o mucho, los pequeños se inhibieron.

La creación de historias requiere elegir personajes, construir tramas y resolver conflictos, y este ejercicio narrativo supuso una ocasión perfecta para impulsar su capacidad para describir y/o idear de forma sencilla personajes. Sin embargo, un 32,1\% la activó algo, un $48,1 \%$ lo hizo bastante, y tan solo un $18,5 \%$ mucho. Lógicamente, se trata de una actividad cognitiva de orden superior que implica un nivel madurativo adecuado para favorecer la asigna-

Tabla 1. Distribución porcentual del alumnado de Infantil según el desarrollo alcanzado en la competencia comunicativa con la elaboración de DST, a juicio del profesorado

\begin{tabular}{|c|c|c|c|c|c|}
\hline Indicadores que definen la Competencia Comunicativa en Educación Infantil & $\begin{array}{c}1 \\
\text { (Poco) }\end{array}$ & $\begin{array}{c}2 \\
\text { (Algo) }\end{array}$ & $\begin{array}{c}3 \\
\text { (Bastante) }\end{array}$ & $\begin{array}{c}4 \\
\text { (Mucho) }\end{array}$ & $\begin{array}{c}\text { No } \\
\text { procede }\end{array}$ \\
\hline I1: Le interesan las aplicaciones digitales para el aprendizaje lecto-escritor & $1,2 \%$ & $23,5 \%$ & $29,6 \%$ & $45,7 \%$ & $0 \%$ \\
\hline I2: Comparte y expresa sus ideas oralmente & $1,2 \%$ & $30,9 \%$ & $34,6 \%$ & $33,3 \%$ & $0 \%$ \\
\hline I3: Se expresa clara y coherentemente, con vocabulario acorde a su edad & $4,9 \%$ & $24,7 \%$ & $35,8 \%$ & $34,6 \%$ & $0 \%$ \\
\hline I4: Pronuncia y entona adecuadamente & $6,2 \%$ & $24,7 \%$ & $34,6 \%$ & $34,6 \%$ & $0 \%$ \\
\hline I5: Reconoce y utiliza los elementos propios del lenguaje escrito & $9,9 \%$ & $25,9 \%$ & $35,8 \%$ & $28,4 \%$ & $0 \%$ \\
\hline I6: Narra una historia oralmente con ayuda & $0 \%$ & $25,9 \%$ & $44,4 \%$ & $29,6 \%$ & $0 \%$ \\
\hline I7: Participa en la creación de la narración colaborativamente & $1,2 \%$ & $38,3 \%$ & $34,6 \%$ & $25,9 \%$ & $0 \%$ \\
\hline I8: Es capaz de describir y/o idear de forma sencilla personajes & $1,2 \%$ & $32,1 \%$ & $48,1 \%$ & $18,5 \%$ & $0 \%$ \\
\hline I9: Comprende las nociones espacio-temporales para crear historias & $3,7 \%$ & $16 \%$ & $44,4 \%$ & $21 \%$ & $14,8 \%$ \\
\hline I10: Comprende y muestra interés por narraciones audiovisuales sencillas & $0 \%$ & $17,3 \%$ & $29,6 \%$ & $53,1 \%$ & $0 \%$ \\
\hline
\end{tabular}


Tabla 2. Media y desviación típica en relación a la competencia comunicativa desarrollada por el alumnado de Educación Infantil

\begin{tabular}{lcc}
\hline Indicadores que definen la Competencia Comunicativa en Educación Infantil & Media & \multicolumn{1}{c}{ Desviación típica } \\
\hline I1: Le interesan las aplicaciones digitales para la lecto-escritura & 3,20 & 3,00 \\
I2: Comparte y expresa sus ideas oralmente & 3,00 & 0,84 \\
I3: Se expresa clara y coherentemente, con vocabulario acorde a su edad & 2,98 & 0,83 \\
I4: Pronuncia y entona adecuadamente & 2,83 & 0,89 \\
I5: Reconoce y utiliza los elementos propios del lenguaje escrito & 3,04 & 0,92 \\
I6: Narra una historia oralmente con ayuda & 2,85 & 2,84 \\
I7: Participa en la creación de la narración colaborativamente & 2,53 & 0,74 \\
I8: Es capaz de describir y/o idear de forma sencilla personajes & 3,36 \\
I9: Comprende las nociones espacio-temporales para crear historias & 0,73 \\
I10: Comprende y muestra interés por narraciones audiovisuales sencillas & 0,76 \\
\hline
\end{tabular}

ción de cualidades físicas y psicológicas a los protagonistas de la historia ideada.

Otra habilidad potenciada con esta práctica es la capacidad de comprender las nociones espacio-temporales que definen el relato, tales como el antes y el después de una acción, así como para ubicar el momento en el que se desarrolla, elementos que mayoritariamente adquirieron bastante los alumnos de Educación Infantil (44,4\%), y mucho un $21 \%$. Sin embargo, con los más pequeños se realizaron relatos en los que, para reducir su complejidad, no se precisaba de estos matices. Por ello, un $14,8 \%$ no fue evaluado a ese respecto (Tabla 1 ).

En la Tabla 2 se presentan las medias relativas a cada uno de los indicadores que contribuyen a explicar la competencia comunicativa con objeto de identificar los que se desarrollaron en mayor medida al participar en la práctica de narración digital.

A pesar de que todos los indicadores reflejan valoraciones altas al concluir la experiencia, se observa que la actividad suscitó una gran motivación plasmada en su alto nivel de comprensión e interés manifestado por narraciones audiovisuales sencillas, junto a su interés por la utilización de aplicaciones digitales para facilitar el aprendizaje de la lecto-escritura. También sirvió para potenciar su participación en la creación colaborativa de los relatos y, casi en la misma medida, ha contribuido a favorecer que los menores describan e ideen personajes sencillos.

Si bien no se han encontrado diferencias en las valoraciones de los sujetos de Infantil en función de la variable curso, al efectuar el contraste de medias atendiendo a la variable género se comprueba que los ítems que registran una diferencia estadísticamente significativa son: I3 ( $p=0,024), I 4(p=0,029)$, I7 $(p=0,028)$, I9 $(p=0,034) ; y, I 10(p=0,014)$, todos ellos a favor de las niñas.

\section{Educación primaria}

De manera semejante, el profesorado implicado evaluó a los alumnos de primaria (Tabla 3 ) que participaron en el Proyecto CINEMA, si bien los diez indicadores que perfilan la competencia comunicativa se adaptaron a su desarrollo madurativo.

La participación del alumnado de primaria en la creación colaborativa de DST ha contribuido a activar tanto habilidades lingüísticas, relacionadas con el dominio del idioma y sus reglas básicas, como narrativas, al plantear una historia a partir de un guión protagonizado por personajes ubicados en escenarios determinados. Así, se constata que han activado bastante la mayoría de aspectos que explican la competencia comunicativa (Tabla 3 ).

Tabla 3. Distribución porcentual del alumnado de primaria según el desarrollo alcanzado en la competencia comunicativa con la elaboración de DST, a juicio del profesorado

\begin{tabular}{|c|c|c|c|c|c|}
\hline Indicadores que definen la Competencia Comunicativa en Educación Primaria & $\begin{array}{c}1 \\
\text { (Poco) }\end{array}$ & $\begin{array}{c}2 \\
\text { (Algo) }\end{array}$ & $\begin{array}{c}3 \\
\text { (Bastante) }\end{array}$ & $\begin{array}{c}4 \\
\text { (Mucho) }\end{array}$ & No procede \\
\hline $\begin{array}{l}\text { I1: Elabora composiciones escritas (diálogos, voz en off, etc.) para contextos } \\
\text { digitales }\end{array}$ & $6,5 \%$ & $25,9 \%$ & $40,8 \%$ & $26,9 \%$ & $0 \%$ \\
\hline I2: Utiliza vocabulario, ortografía, puntuación, etc. adecuados a su nivel & $7,5 \%$ & $20,9 \%$ & $44,8 \%$ & $26,9 \%$ & $0 \%$ \\
\hline I3: Selecciona los tiempos verbales correspondientes a la acción correctamente & $4 \%$ & $24,4 \%$ & $41,8 \%$ & $29,4 \%$ & $0,5 \%$ \\
\hline I4: Adopta un registro lingüístico acorde con la narración & $7,5 \%$ & $24,9 \%$ & $41,8 \%$ & $24,9 \%$ & $1 \%$ \\
\hline $\begin{array}{l}\text { I5: Manifiesta una dicción, pronunciación y entonación adecuada en diálogos o } \\
\text { voz en off }\end{array}$ & $9 \%$ & $24,4 \%$ & $35,8 \%$ & $25,4 \%$ & $5,5 \%$ \\
\hline I6: Selecciona y planifica una historia siguiendo un guión preestablecido & $8 \%$ & $20,4 \%$ & $46,3 \%$ & $25,4 \%$ & $0 \%$ \\
\hline I7: Construye relatos con coherencia interna (presentación, nudo, desenlace) & $9 \%$ & $23,4 \%$ & $42,3 \%$ & $24,9 \%$ & $0,5 \%$ \\
\hline I8: Idea personajes y planifica adecuadamente escenarios y discursos & $7 \%$ & $27,4 \%$ & $40,8 \%$ & $24,4 \%$ & $0,5 \%$ \\
\hline I9: Comprende y/o expresa la intención comunicativa de su relato & $10,4 \%$ & $32,8 \%$ & $35,8 \%$ & $19,9 \%$ & $1 \%$ \\
\hline I10: Dota al relato del ritmo apropiado mediante la interacción entre personajes & $10 \%$ & $29,4 \%$ & $34,8 \%$ & $20,9 \%$ & $5 \%$ \\
\hline
\end{tabular}


Tabla 4. Media y desviación típica en relación a la competencia comunicativa desarrollada por los alumnos de Educación Primaria

\begin{tabular}{lcc}
\hline Indicadores que definen la Competencia Comunicativa en Educación Primaria & Media & Desviación típica \\
\hline I1: Elabora composiciones escritas (diálogos, voz en off, ...) para contextos digitales & 2,88 & 0,88 \\
I2: Utiliza vocabulario, ortografía, puntuación, etc. adecuados a su nivel & 2,91 & 2,96 \\
I3: Selecciona los tiempos verbales correspondientes a la acción correctamente & 2,82 & 0,87 \\
I4: Adopta un registro lingüístico acorde con la narración & 2,67 & 2,89 \\
I5: Manifiesta una dicción, pronunciación y entonación adecuada en diálogos & 2,82 & 0,92 \\
I6: Selecciona y planifica una historia siguiendo un guión preestablecido & 2,82 \\
I7: Construye relatos con coherencia interna (presentación, nudo, desenlace) & 2,11 \\
I8: Idea personajes y planifica adecuadamente escenarios y discursos & 0,87 \\
I9: Comprende y/o expresa la intención comunicativa de su relato & 2,57 \\
I10: Dota al relato del ritmo apropiado mediante la interacción entre personajes & 0,90 & 0,95 \\
\hline
\end{tabular}

Más de la cuarta parte los ha activado mucho: en concreto, un $29,4 \%$ selecciona correctamente los tiempos verbales, un aproximado $27 \%$ utiliza vocabulario, ortografía, puntuación, etc. adecuados a su nivel, y otro tanto sabe elaborar composiciones escritas (diálogos, voz en off, etc.) adaptadas a los contextos digitales.

El 46,3\% es capaz de seleccionar y planificar historias siguiendo un guión con bastante destreza, el $44,8 \%$ utiliza vocabulario, ortografía, puntuación, etc. bastante adecuado. Mientras, el 43,2\% comprende algo o poco y/o sabe expresar de forma limitada la intención comunicativa de su relato, otro $39,4 \%$ no sabe dotar del ritmo apropiado a su relato mediante la interacción entre personajes, o lo hace de forma precaria, y al 27,14\% le cuesta idear personajes y planificar escenarios y discursos, sobre todo en los cursos inferiores.

Por otro lado, y dado que en la mayoría de los relatos los propios alumnos dieron vida a los personajes con sus voces, se observa que -según sus profesores- el 35,8\% presenta bastantes progresos en su dicción, pronunciación y entonación al locutar los diálogos o voz en off de sus historias, si bien son los mayores los que lógicamente muestran un avance mayor (25,4\%). El 42,3\% demuestra construir relatos con bastante coherencia interna, y además, casi un $25 \%$ lo hace con mucha destreza, identificando la presentación, el nudo y el desenlace. Igualmente sucede respecto a su habilidad para adoptar el registro lingüístico más acorde con su narración.

La Tabla 4 muestra las medias registradas en cada indicador que ha servido para evaluar los progresos individuales del alumnado al concluir la experiencia narrativa en formato digital.

Con medias muy semejantes se presentan los logros alcanzados por los alumnos de primaria en cada uno de los indicadores que integran la competencia comunicativa, siendo perceptibles los relativos a la correcta asignación de los tiempos verbales, seguidos de los avances en el adecuado uso del vocabulario, ortografía y puntuación. Con los contrastes posteriores no se detectan diferencias en el alumnado de primaria según la variable sexo; sin embargo, al efectuar el contraste de medias atendiendo a la variable nivel educativo se comprueba que los ítems que registran diferencias estadísticamente significativas son: I1 $(p=0,017), I 2(p=0,037), I 3$ $(p=0,014), I 6(p=0,035), I 7(p=0,026), I 8(p=0,002), I 9(p=0,000)$, para los sujetos del nivel inicial y el superior de primaria, a favor lógicamente de los de cursos superiores.

\subsection{Evaluación de la competencia digital activada con los rela- tos digitales}

\section{Educación Infantil}

La competencia digital en Educación Infantil ha sido definida y valorada a partir de cinco indicadores ligados directamente al diseño de DST. Los resultados obtenidos por los alumnos de las escuelas rurales participantes en el proyecto CINEMA revelan las habilidades ejercitadas con la creación de las producciones diseñadas (Tabla 5).

Se constata el gran interés mostrado por los niños/as de Educación Infantil por las herramientas digitales utilizadas para comunicarse y aprender de una manera lúdica, pues el $42 \%$ presenta mucho interés y un 38,3\% bastante. Cabe destacar que el 31\% utiliza bastante bien las aplicaciones informáticas para crear relatos y un $18,5 \%$ muestra un alto nivel de manejo. No obstante, el 46,7\% del alumnado de Infantil no fue evaluado por no tener las capacidades y habilidades necesarias para manejar estas herramientas, dada su corta edad.

Tabla 5. Distribución porcentual del alumnado de Infantil según el desarrollo alcanzado en la competencia digital con la elaboración de DST, a juicio del profesorado

\begin{tabular}{|c|c|c|c|c|c|}
\hline Indicadores que definen la Competencia Digital en Educación Infantil & $\begin{array}{c}1 \\
\text { (Poco) }\end{array}$ & $\begin{array}{c}2 \\
\text { (Algo) }\end{array}$ & $\begin{array}{c}3 \\
\text { (Bastante) }\end{array}$ & $\begin{array}{c}4 \\
\text { (Mucho) }\end{array}$ & No procede \\
\hline I1: Maneja de forma elemental los soportes tecnológicos utilizados & $0 \%$ & $24,7 \%$ & $49,4 \%$ & $25,9 \%$ & $0 \%$ \\
\hline $\begin{array}{l}\text { I2: Muestra interés por las herramientas digitales para comunicarse, jugar y } \\
\text { aprender }\end{array}$ & $0 \%$ & $19,8 \%$ & $38,3 \%$ & $42 \%$ & $0 \%$ \\
\hline $\begin{array}{l}\text { I3: Emplea de forma básica las aplicaciones informáticas para la creación de } \\
\text { los relatos }\end{array}$ & $1,2 \%$ & $3,7 \%$ & $30,9 \%$ & $18,5 \%$ & $46,7 \%$ \\
\hline I4: Utiliza los medios tecnológicos adecuadamente & $0 \%$ & $18,5 \%$ & $54,3 \%$ & $25,9 \%$ & $1,2 \%$ \\
\hline I5: Explora las posibilidades expresivas y creativas de los medios digitales & $0 \%$ & $27,2 \%$ & $38,3 \%$ & $30,9 \%$ & $3,7 \%$ \\
\hline
\end{tabular}


Tabla 6. Media y desviación típica en relación a la competencia digital desarrollada por los alumnos de Educación Infantil

\begin{tabular}{lcc}
\hline Indicadores que definen la Competencia Digital en Educación Infantil & Media & Desviación típica \\
\hline I1: Maneja de forma elemental los soportes tecnológicos utilizados & 3,01 & 0,71 \\
I2: Muestra interés por herramientas digitales para comunicarse, jugar y aprender & 3,22 \\
I3: Emplea de forma básica aplicaciones informáticas para la creación de los relatos & 1,75 \\
I4: Utiliza los medios tecnológicos adecuadamente & 1,69 & 3,04 \\
I5: Explora las posibilidades expresivas y creativas de los medios digitales & 0,74 & 2,93 \\
\hline
\end{tabular}

La creación de DST implica diferentes tareas: toma de imágenes, grabación de locuciones, etc. Ello sirvió para constatar que el 49,4\% del alumnado de Educación Infantil maneja bastante los soportes tecnológicos y un 25,9\% mucho, junto al 54,3\%, que utiliza dichos medios de manera bastante adecuada y un $25,9 \%$ con pericia (Tabla 5). Algunos de los relatos fueron adaptaciones de cuentos y otros historias originales creadas por los alumnos, lo que les brindó la oportunidad de explorar las posibilidades expresivas y creativas de los medios digitales: según el profesorado, un 38,3\% lo desarrolló bastante bien, y un 30,9\% muy adecuadamente (Tabla 5), permitiéndoles aproximarse a la producción audiovisual, gestando un relato, ideando personajes y escenarios, locutándolo, etc.

En la Tabla 6 se identifican las medias obtenidas para el indicador que explica la competencia digital desarrollada por el alumnado de Infantil al diseñar sus relatos.

En la mayoría de los indicadores se obtienen puntuaciones altas, destacando especialmente el interés mostrado por los menores hacia el uso de herramientas digitales para la comunicación y el aprendizaje lúdico, seguida del uso adecuado de los medios tecnológicos. Sin embargo, se puede apreciar que en lo referente al uso de aplicaciones informáticas para la creación de relatos, la media está muy por debajo del resto. Las únicas diferencias estadísticamente significativas identificadas, con el posterior contraste de medias, han sido en función del sexo para el ítem 4: utiliza los medios tecnológicos adecuadamente $(\mathrm{p}=0,025)$, cuyas puntuaciones mayores las reciben las niñas.

\section{Educación Primaria}

Igualmente, para educación primaria se definieron otros cinco indicadores, adaptados a esta etapa educativa, para valorar el nivel de competencia digital alcanzado al diseñar los DST (Tabla 7).

El 48,8\% utiliza algo programas sencillos para la edición audiovisual y un $19,4 \%$ bastante, pues en los primeros cursos fueron los docentes los que realizaron el montaje y producción de los relatos. Un $40 \%$ muestra solo algunos conocimientos del lenguaje audiovisual (planos utilizados, movimientos de cámara...), y un
$31,8 \%$ lo desarrolló bastante. Casi un 22\% no fue evaluado pues no efectuó esa tarea. El 42,8\% conoce algo la técnica elegida para desarrollar los DST y un 31,8\% bastante. De los siete relatos diseñados colaborativamente, 4 optaron por la grabación de vídeo, dos eran fotomontajes y otro adoptó la técnica del stop-motion. Un 34,3\% colaboró algo en la búsqueda, selección y elaboración de recursos, un $30,3 \%$ ayudó bastante y solo un $14,4 \%$ colaboró mucho (Tabla 7 ). Un 20,9\% desconoce cómo ensamblar imagen-locución, mientras que el 37,3\% posee alguna noción. Los más pequeños no fueron valorados, pues el proceso de montaje lo realizó el profesorado.

A continuación, se analizan las medias de los indicadores que definen la competencia digital (Tabla 8).

Las medias evidencian que apenas existen variaciones entre los indicadores analizados. La colaboración activa para la búsqueda, selección y elaboración de recursos fue desarrollada algo más que el resto, seguidas por los conocimientos básicos del lenguaje audiovisual, mientras que la habilidad menos activada fue el ensamblaje de imagen y locución, efectuado por el profesorado especialmente en los primeros cursos de primaria. No existen diferencias por sexo, pero sí en función del nivel, destacando con mejores puntuaciones los sujetos de los cursos superiores para los indicadores: I1 $(p=0,026), I 3(p=0,008), I 4(p=0,000)$, y I5 $(p=0,005)$.

\section{Discusión y conclusiones}

A la vista de los resultados, se puede afirmar que el profesorado implicado en el proyecto ha observado y detectado que el alumnado de Educación Infantil ha desarrollado con la creación de los relatos digitales habilidades de uso de la lengua oral y de la iniciación en la lengua escrita, además de progresar en el conocimiento y manejo de las formas narrativas audiovisuales, y en la producción de narraciones orales, además de ejercitar habilidades específicas vinculadas a la adquisición de códigos narrativos. Además, se ha observado que, entre el alumnado de Educación Infantil, las niñas destacan significativamente en su capacidad para expresarse de forma más clara y coherente, uso de vocabu-

Tabla 7. Distribución porcentual de los alumnos en función de la evaluación efectuada por el profesorado de Educación Primaria en relación a la competencia digital

\begin{tabular}{|c|c|c|c|c|c|}
\hline Indicadores que definen la Competencia Digital en Educación Primaria & $\begin{array}{c}1 \\
\text { (Poco) }\end{array}$ & $\begin{array}{c}2 \\
\text { (Algo) }\end{array}$ & $\begin{array}{c}3 \\
\text { (Bastante) }\end{array}$ & $\begin{array}{c}4 \\
\text { (Mucho) }\end{array}$ & No procede \\
\hline I1: Conoce la técnica elegida para desarrollar los relatos digital & $5,5 \%$ & $42,8 \%$ & $31,8 \%$ & $9,5 \%$ & $10,4 \%$ \\
\hline $\begin{array}{l}\text { I2: Conoce los elementos básicos del lenguaje audiovisual (tipo de planos, } \\
\text { movimientos cámara) }\end{array}$ & $12,4 \%$ & $40,8 \%$ & $20,4 \%$ & $4,5 \%$ & $21,9 \%$ \\
\hline $\begin{array}{l}\text { I3: Colabora activamente en la búsqueda, selección y elaboración de recursos } \\
\text { audiovisuales }\end{array}$ & $3,5 \%$ & $34,3 \%$ & $30,3 \%$ & $14,4 \%$ & $17,4 \%$ \\
\hline I4: Aplica programas sencillos para la edición audiovisual & $11,4 \%$ & $48,8 \%$ & $19,4 \%$ & $5 \%$ & $15,4 \%$ \\
\hline I5: Conoce cómo se realiza el ensamblaje imagen-locución (voz en off) & $20,9 \%$ & $37,3 \%$ & $12,4 \%$ & $2,5 \%$ & $26,9 \%$ \\
\hline
\end{tabular}


Tabla 8. Media y desviación típica en relación a la competencia digital desarrollada por los alumnos de Educación Primaria

\begin{tabular}{|c|c|c|}
\hline Indicadores que definen la Competencia Digital en Educación Primaria & Media & Desviación típica \\
\hline I1: Conoce la técnica elegida para desarrollar los relatos digital & 2,88 & 0,88 \\
\hline I2: Conoce los elementos básicos del lenguaje audiovisual & 2,91 & 0,87 \\
\hline I3: Colabora activamente en la búsqueda, selección y elaboración de recursos audiovisuales & 2,96 & 0,86 \\
\hline I4: Aplica programas sencillos para la edición audiovisual & 2,82 & 0,92 \\
\hline I5: Conoce cómo se realiza el ensamblaje imagen-locución (voz en off) & 2,67 & 1,11 \\
\hline
\end{tabular}

lario y pronunciación, así como en aspectos relacionados con el discurso narrativo.

En Primaria, se ha observado que con esta actividad se ha mejorado la competencia comunicativa oral y escrita de todo el alumnado, y ha familiarizado a los estudiantes con modelos textuales destinados a la creación audiovisual y con los códigos narrativos. El alumnado ha encontrado más dificultades en el manejo del ritmo narrativo, el control de la articulación y los códigos paralingüísticos en la locución del texto y la adecuación del mensaje a la intencionalidad. Aunque los resultados han sido bastante satisfactorios, los estudiantes de los cursos superiores destacan en la elaboración de composiciones escritas (diálogos, voz en off, etc.), uso de vocabulario, ortografía, puntuación, utilización de tiempos verbales, capacidad de idear y desarrollar una historia coherente y bien estructurada, creación de personajes consistentes y planificación de escenarios y discursos. También demuestran comprender y/o expresar la intención comunicativa de sus relatos mejor que el alumnado de los primeros cursos de primaria, divergencias en nivel de competencia comunicativa que parecen lógicas dada la diferencia de edad.

En cuanto a competencia digital, se destaca el interés del Alumnado de Infantil por el uso de las herramientas tecnológicas. Aunque en muchos casos los docentes -o el alumnado de primaria, en las experiencias multinivel- han asumido las tareas más complejas, relativas al manejo de herramientas y aplicaciones informáticas, y la participación del alumnado de Educación Infantil en este proyecto ha favorecido el desarrollo de su competencia digital a un nivel básico. Además, se han detectado diferencias estadísticamente significativas en función del sexo, constatándose que las niñas de esta etapa utilizan los medios tecnológicos mejor que los niños al servicio de la creación de los relatos digitales.

En primaria, el alumnado se ha iniciado en el lenguaje audiovisual y en el conocimiento de las técnicas y herramientas para la creación de DST, destacando los resultados en búsqueda, selección y elaboración de recursos para la creación audiovisual, conocimientos de lenguaje audiovisual y conocimiento de la técnica empleada. Sin embargo, no todo el alumnado ha participado por igual en el montaje técnico, ya que en los cursos inferiores el profesorado ha asumido las tareas más complejas, como el ensamblaje de imagen y sonido. Finalmente, se aprecian diferencias respecto a la variable nivel, obteniendo mejores valoraciones los sujetos de los cursos superiores en conocimiento de las técnicas elegidas para desarrollar los relatos digitales, empleo básico de aplicaciones informáticas, aplicación de programas sencillos para la edición audiovisual, y conocimiento de los procedimientos de ensamblaje imagen-locución.

En conclusión, esta experiencia ha contribuido al desarrollo de habilidades comunicativas orales y escritas y, en particular, al manejo de los códigos del relato aplicados a la creación en soporte multimedia. Asimismo, se ha observado una mejora en la competencia digital del alumnado, favoreciendo la iniciación en el conocimiento y manejo de recursos tecnológicos diversos, según la técnica utilizada en cada caso.

Además, las características propias de los centros rurales han hecho posible el desarrollo de proyectos multinivel, en los cuales los estudiantes han trabajado colaborativamente en la creación de DST, asumiendo en cada caso tareas acordes a su edad y capacidades. También se ha favorecido la colaboración del profesorado de los diferentes cursos en la realización de proyectos conjuntos, algo que puede incidir favorablemente en el desarrollo de la cultura innovadora con TIC en las escuelas rurales (Del Moral, Villalustre y Neira, 2014), de forma similar a lo que señalan Bidwell, Reitmaier, Marsden y Hansen (2010). Por último, los DST se han revelado como unos recursos idóneos para llevar a cabo proyectos complejos e interdisciplinares, aptos para desarrollar diferentes competencias, de las que cabe destacar su contribución a la comunicativa y la digital, dada la multiplicidad de lenguajes y tareas comunicativas que permiten, así como la pluralidad de herramientas tecnológicas que implican, como apuntan Fletcher y Cambre (2009). Además, son lo suficientemente versátiles como para adaptarse a los diferentes niveles y etapas educativas y, por último, ofrecen interesantes posibilidades para la innovación con TIC en el ámbito rural.

Como limitaciones de la investigación cabe señalarse que habría que depurar algunos de los aspectos metodológicos para triangular las valoraciones del progreso competencial del alumnado, así como controlar la variable desarrollo evolutivo propio del alumnado, pues ha podido contribuir a constatar unos resultados mejores en los cursos superiores.

Entre las líneas de investigación futuras se encuentra la generación de proyectos narrativos interdisciplinares para crear DST que potencien la creatividad y las competencias emocionales ligadas al diseño colaborativo entre los escolares.

\section{Referencias bibliográficas}

Area, M. (2010). El proceso de integración y uso pedagógico de las TIC en los centros educativos. Un estudio de casos. Revista de Educación, 352, 77-97.

Area, M., y Ribeiro, M. T. (2012). De lo sólido a lo líquido: las nuevas alfabetizaciones ante los cambios culturales de la Web 2.0. Comunicar, 19(38), 13-20. http://dx.doi.org/10.3916/ C38-2012-02-01

Banaszewski, T. (2002). Digital storytelling finds its place in the classroom. Multimedia Schools, 9(1), 32-35.

Bidwell, N.J., Reitmaier, T., Marsden, G., y Hansen, S. (2010, April). Designing with mobile digital storytelling in rural Africa. In Proceedings of the Conference on Human Factors in Computing Systems (SIGCHI) (pp. 1593-1602). New York: ACM. doi_10.1145/1753326.1753564

Burke, Q., y Kafai, Y. B. (2012, February). The writers' workshop for youth programmers: digital storytelling with scratch in middle school classrooms. In Proceedings of the 43rd ACM 
Technical Symposium on Computer Science Education (pp. 433438). New York: ACM.

Calvani, A., Ranieri, M., y Fini, A. (2010). Digital Competence in K-12: theoretical models, assessment tools and empirical research. Anàlisi: Quaderns de Comunicació i Cultura, 40, 157-171.

Canale, M. (1983). De la competencia comunicativa a la pedagogía comunicativa del lenguaje. En M. Llobera (coord.) (1995). Competencia comunicativa. Documentos básicos en la enseñanza de lenguas extranjeras (pp. 63-83). Madrid: Edelsa.

Clayton, M. J. (1997). Delphi: A techique to armes expert opinión for criticas decisión-marking tasks in education. Education Psycology, 17(4), 373-386.

Del Moral, M. E., Villalustre, L. y Neira, M. R. (2014a). Oportunidades de las TIC para la innovación educativa en las escuelas rurales de Asturias. Aula Abierta, 42, 61-67.

Del Moral, M. E., Villalustre, L. y Neira, M. R. (2014b). Variables asociadas a la cultura innovadora con TIC en escuelas rurales. Profesorado. Revista de Currículo y Formación del Profesorado, 18(3), 9-25.

Del Moral, M.E., Villalustre, L. y Neira, M.R. (2016). Instruments to Measure Competences in Digital Storytelling Design at Preschool and Primary Education. International Journal of Technologies in Learning (IJTL), 23(3), 21-40. DOI: 10.18848/23270144/CGP/v23i03

De Pablos, J., Colás, P., y González, T. (2010). Factores facilitadores de la innovación con TIC en los centros escolares. Un análisis comparativo entre diferentes políticas educativas autonómicas. Revista de Educación, 352, 23-51.

Dettori, G., y Paiva, A. (2009). Narrative learning in technology-enhanced environments. In: N. Balacheff et al. (ed.), Technology-Enhanced Learning (pp. 55-69). Netherlands: Springer.

Dobson, S. (2005). Narrative competence and the enhancement of literacy. Some theoretical reflections. International Journal of Media, Technology and Lifelong Learning, 1(2), 1-14.

Figg, C., McCartney, R., y Gonsoulin, W. (2010). Impacting academic achievement with student learners teaching digital storytelling to others: The ATTTCSE digital video project. Contemporary Issues in Technology and Teacher Education, 10(1), 38-79.

Fletcher, C., y Cambre, C. (2009). Digital storytelling and implicated scholarship in the classroom. Journal of Canadian Studies, 43(1), 109-130.

Frazel, M. (2010). Digital storytelling guide for educators. Washington, DC: International Society for Technology in Education.

Gazarian, P. K. (2010). Digital stories: Incorporating narrative pedagogy. Journal of Nursing Education, 49(5), 287-290. doi: 10.3928/01484834-20100115-07

Guo, R. X., Dobson, T., y Petrina, S. (2008). Digital natives, digital immigrants: An analysis of age and ICT competency in teacher education. Journal of Educational Computing Research, 38(3), 235-254. doi: 10.2190/EC.38.3.a

Hatlevik, O. E., y Christophersen, K. A. (2013). Digital competence at the beginning of upper secondary school: Identifying factors explaining digital inclusion. Computers y Education, 63, 240-247. doi:10.1016/j.compedu.2012.11.015

Heo, M. (2009). Digital storytelling: An empirical study of the impact of digital storytelling on pre-service teachers' self-efficacy and dispositions towards educational technology. Journal of Educational Multimedia and Hypermedia, 18(4), 405-428.

Hung, H-L., Altschuld, J. W., y Lee, Y. (2008). Methodological and conceptual issues confronting a cross-country Delphi study of educational program evaluation. Evaluation and Program Planning, 31, 191-198.

Instituto Cervantes (2002). Marco Común Europeo de referencia para las lenguas: aprendizaje enseñanza, evaluación. Madrid: MECD/
Anaya. Recuperado de http://cvc.cervantes.es/ensenanza/biblioteca ele/marco/

ITE (2011). Competencia digital. Recuperado de http://recursostic. educacion.es/blogs/europa/media/blogs/europa/informes/ Competencia_Digital_Europa_ITE_marzo_2011.pdf

Janssen, J., Stoyanov, S., Ferrari, A., Punie, Y., Pannekeet, K., y Sloep, P. (2013). Experts' views on digital competence: Commonalities and differences. Computers y Education, 68, 473481. doi:10.1016/j.compedu.2013.06.008

Krumsvik, R. J. (2014). Teacher educators' digital competence. Scandinavian Journal of Educational Research, 58(3), 269-280. doi: 10.1080/00313831.2012.726273

Lambert, J. (2010). Cookbook for Digital Storytelling. New York: Digital Diner Press.

Lai, K. W. (2011). Digital technology and the culture of teaching and learning in higher education. Australasian Journal of Educational Technology, 27(8), 1263-1275. doi: http://dx.doi. org/10.14742/ajet.v27i8.892

Lomas, C. (2015). Fundamentos para una enseñanza comunicativa del lenguaje. En C. Lomas (coord.). Fundamentos para una enseñanza comunicativa del lenguaje (pp. 9-23). Barcelona: Graó.

Lowenthal, P. (2009). Digital Storytelling in Education. An Emerging Institutional Technology? In J. Hartley y $\mathrm{K}$. McWilliam (eds.), Story Circle: Digital Storytelling around the World (pp. 252-259). Oxford, UK: Wiley-Blackwell. doi: 10.1002/9781444310580.ch18

Malita, L., y Martin, C. (2010). Digital storytelling as web passport to success in the 21st century. Procedia-Social and Behavioral Sciences, 2(2), 3060-3064. doi:10.1016/j.sbspro.2010.03.465

Mihailidis, P., y Cohen, J. (2013). Exploring curation as a core competency in digital and media literacy education. Journal of Interactive Media in Education, 1, 1-19. http://doi. org/10.5334/2013-02

Ohler, J. B. (2013). Digital storytelling in the classroom: New media pathways to literacy, learning, and creativity. California: Corwin Press.

Orden ECD/65/2015, por la que se describen las relaciones entre las competencias, los contenidos y los criterios de evaluación de la educación primaria, la educación secundaria obligatoria y el bachillerato de 21 de enero, BOE núm. 25, 44.037- 44.048 (2015).

Parlamento Europeo y Consejo (2006). Recomendación 2006/962/ CE de 18 de diciembre de 2006 del Parlamento Europeo y del Consejo sobre las competencias clave para el aprendizaje permanente. Diario Oficial L 394 de 30.12.2006.

Robin, B. (2008). Digital Storytelling: A Powerful Technology Tool for the 21st Century Classroom. Theory Into Practice, 47(3), 220-228. doi: 10.1080/00405840802153916

Rodríguez-Illera, J.L. y Escofet, A. (2006). Aproximación centrada en el estudiante como productor de contenidos digitales en cursos híbridos. RUSC: Revista de Universidad y Sociedad del Conocimiento, 2(3), 20-28. Recuperado de www.uoc.edu/ rusc/3/2/dt/esp/rodriguez escofet.pdf

Skouge, J. R., y Rao, K. (2009). Digital Storytelling in Teacher Education: Creating Transformations through Narrative. Educational Perspectives, 42, 54-60.

Valverde, J., Garrido, M. C., y Sosa, M. J. (2010). Políticas educativas para la integración de las TIC en Extremadura y sus efectos sobre la innovación didáctica y el proceso enseñanza-aprendizaje: la percepción del profesorado. Revista de Educación, 352, 99-124.

Villalustre, L. y Del Moral, M. E. (2014). Digital storytelling: una nueva estrategia para narrar historias y adquirir competencias por parte de los futuros maestros. Revista Complutense de Educación, 25(1), 115-132. 